\title{
This issue of Acta
}

doi: 10.1111/j.1755-3768.2008.01177.x

This issue of Acta resumes the original name of the journal, Acta Ophthalmologica.

The cover figure shows the development of drusen of the optic nerve head over 23 years, as observed by Lars Frisén in Gothenburg and reported in this issue of Acta.

The guest editorial is written by Kerstin Wickström in Uppsala and discusses antibiotic treatment in acute bacterial conjunctivitis.

\section{Review article}

Gunnar Høvding, in Bergen, reviews acute bacterial conjunctivitis, which is one of the most common eye diseases seen by general practitioners and is seen frequently in most ophthalmologists' offices. Høvding reviews the epidemiology, aetiology, clinical features, complications, differential diagnosis and therapy of acute bacterial conjunctivitis.

\section{Retina}

Kyhn et al., in Copenhagen, created retinal holes and detachments in pigs and concluded that it is feasible to access the subretinal space in pigs without causing permanent changes in the multifocal electroretinogram. This is important in the context of subretinal surgery and transplantation.

Holmgaard et al., in Århus, examined the vascular contractility of porcine retinal arterioles and found this to be inhibited by perivascular retinal tissue. This process involves $\mathrm{N}$ methyl-D-aspartic acid receptors, gamma-aminobutyric acid agonists and the cyclo-oxygenase enzyme.

Soliman et al., in Copenhagen and Egypt, find a correlation between changes in optical coherence tomography (OCT) and fluorescein angiography in diabetic macular oedema. They point out that each method has advantages: the fluorescein angiography may see early changes better than the OCT, whereas serous detachment of the fovea is seen only with the OCT and not on fluorescein angiography. Therefore, the combination of these techniques is useful in grading diabetic macular oedema.

Margolis et al., from the USA, contribute a case series where they use intravitreal triamcinolone to reduce the risk of increased macular oedema following panretinal photocoagulation in diabetic patients.

\section{Cornea}

Ehlers et al., in Århus and Tubingen, discuss the phenotypic variability of Meesmann's microcystic dystrophy and present a large Danish family with the disease.

Sohajda et al., in Hungary, compared two recent A-scan ultrasound devices for measurements of corneal thickness, anterior chamber depth and axial length. While each of the instruments is reliable, they do not give exactly the same values and seem not to be interchangeable.

Higaki et al., in Japan, report longterm results from 14 patients who underwent deep lamellar keratoplasty using a graft with its own endothelium; they find this to be a safe and valuable procedure.

\section{Strabismus}

Koch et al., in Austria, examine strabismus correction in patients with high myopia and use biomechanical simulation to improve the accuracy of strabismus correction in these cases.

\section{Uveitis}

Norrsell and Sjödell, in Gothenburg, examined 54 patients with Fuch's heterochromic uveitis. They point out that delay in diagnosis is common and that careful examination of heterochromia, iris atrophy and vitreous opacity is essential for prompt diagnosis.

\section{Cataract}

Sato et al., in Japan, perform a prospective randomized study of the optimization of the use of viscoelastic materials in 'soft-shell techniques'; they find this to be effective in protecting corneal endothelial cells during phacoemulsification.

\section{Glaucoma}

Béchetoille et al., in France, use a 36item questionnaire on health-related quality of life in patients with glaucoma and ocular hypertension. The results of the questionnaire show an excellent correlation with disease progression in glaucoma.

\section{Blood-flow physiology}

Mavroudis et al., in Greece and the USA, examine the reproducibility of Heidelberg retinal flowmetry and find this to be a highly reproducible method in the hands of trained graders. The blood-flow results are independent of the exact placement of the analysis windows and between individual graders.

\section{Oculoplastics}

Hwang et al., in South Korea, recommend lamellar splitting of the lid margin in the surgical repair of epiblepharon.

\section{Pharmacology}

Vehanen et al., in Finland, examine the use of a biopolymer for peribulbar injection and controlled drug release. They found the polymer to be non-toxic and to release the drug over 24 hours.

Kaji et al., in Japan, compare suspensions of triamcinolone acetonite and 11-deoxycortisol to visualize the vitreous body prolapsed into the anterior chamber after posterior capsular rupture in pigs.

\section{Optic nerve}

Nangia et al., in India and Germany, found that the mean optic disc area is larger in an Indian population than in White patients. Axial length and myopic refractive error influence optic disc size.

I hope that all subscribers will find informative and enjoyable material in this issue of Acta.

Einar Stefánsson 